\title{
THE ASSOCIATION OF INDUCED CHANGES IN FLAX
}

\author{
ALAN DURRANT \\ Department of Agricultural Botony, University College of Wales, Aberystwyth
}

Received 28.ii.73

\section{Summary}

The three characters, plant weight, amount of nuclear DNA, and $h$ (hairless septa), in which heritable changes have been induced by the environment, are associated in their induction, in inheritance and in crosses, but the association is not complete. In the $\mathrm{F}_{2}, h$ is associated with plant weight in the direction of linkage while a flower colour mutation, $w$, obtained by irradiating plants with $\mathrm{Co}^{60}$, is associated with plant weight in the opposite direction to linkage. Although $h$ and $w$ segregate independently the amount of "positive linkage" of $h$ with plant weight is inversely related to the amount of "negative linkage" of $w$ with plant weight in the particular environments and crosses used. The three environmentally inducible characters are unstable in the $F_{1}$ and none of the genetic variation in $F_{2}$ need be due to classical segregation or recombination. It is probable that they and $w$ are on the same chromosome and that changes in amount of DNA, which it is supposed are due to changes in number of reiterated sequences, alter the heterochromatisation or structure of the chromosomes and the activity of genes in the region. In heterozygotes the stable equilibria of the homozygotes are destroyed, the induced differences in heterochromatisation of the homologous chromosomes are disrupted giving heterochromatisation of sub-regions, or different amounts of reiteration, whose locations depend upon the intracellular environment

\section{Introduction}

Heritable changes are induced in some flax varieties when they are grown in different environments normally consisting of different fertilisers applied in moderate amounts to compost in a greenhouse with a little supplementary heating. Changes induced in three characters in the variety Stormont Cirrus, a plastic genotroph $(P l)$, are briefly described below (Evans, Durrant and Rees, 1966; Evans, 1968; Durrant and Nicholas, 1970; Durrant, 1962a, 1971; McLellan and Durrant, 1973).

\section{(i) Plant weight}

Nuclear changes giving a large $(L)$ stable genotroph or a small (S) stable genotroph are induced depending on the fertilisers supplied. $L$ is up to five times the size of $S$ and $P l$ is intermediate in weight. $L$ and $S$ breed true and no further changes can be induced in them when grown in different fertilisers. The reciprocal $\mathrm{F}_{1}$ 's of crosses between $L$ and $S$ are intermediate in weight but they are genetically unstable (Durrant, 1962b) giving in the appropriate environments continuous genetic variation which is transmitted to $\mathrm{F}_{2}$ plants. The crosses respond to selection for high and low plant weight and give a range of genetic variation.

\section{(ii) $H$-h character}

$P l$ and $S$ have hairy septa $(H)$ in the capsules, $L$ has hairless septa $(h)$, a change which is induced by the environment at the same time as plant 
weight. Crosses between $L$ and $S$ give $H: h$ ratios of $3: 1$ in the $\mathrm{F}_{2}$ but the heterozygotes are unstable and $H \rightarrow h$ and $h \rightarrow H$ changes occur. Heterozygous, $H h$, plants can be classified into three distinct groups of approximately 30,40 and 50 hairs per septum, compared with 60 per septum for $S$, and those $H h$ plants with higher hair numbers give ratios in the next generation with excess $H$ plants.

\section{(iii) Amount of nuclear $D \mathcal{N A}$}

Changes in amount of nuclear DNA are induced at the same time as the changes in plant weight and $H . \quad L$ has 16 per cent more nuclear DNA than $S ; P l$ has an intermediate amount. The different amounts of DNA are inherited unchanged by later generations provided the plants are grown for the first five weeks in warm conditions, but they will begin to revert towards the intermediate $P l$ value if the plants are grown entirely out-of-doors (Durrant and Jones, 1971). The partial reversion of DNA is not accompanied by any reversion in plant weight. In crosses between $L$ and $S$ the character gives a range of genetic variation in the $\mathrm{F}_{2}$ and it is probably unstable in the $\mathbf{F}_{1}$. Selections for high and low plant weight in the crosses are accompanied by correlated responses in amount of DNA, the high selection lines having more DNA than the low.

A significant association in the direction of linkage was found between plant weight and $H-h$ in the $\mathrm{F}_{2}$ of crosses between $L h$ and $S H$ (Durrant and Nicholas, 1970), supporting the notion that factors for $h$ and plant weight are located near each other on one chromosome. Because of the continuous variation in plant weight in $\mathrm{F}_{2}$ the association is measured by comparing the weight of plants classified according to the relatively clear-cut $H-h$ segregants. Prior to this, similar association studies were made with another factor, a mutation giving white, instead of blue, flowers obtained by irradiating $L$ plants with $\mathrm{Co}^{60}$ and selfing. Plants homozygous for this mutation, $L w / L w$, were similar in appearance to the original $L$ plants, $L W / L W$, except for flower colour. They do not appear to have any chromosomal abnormalities and have the same amount of nuclear DNA as $L W$, i.e. 16 per cent more than $S W . \quad L w$ was crossed with $L W$ and $S W$ to find whether $w$ was associated with plant weight in the $\mathrm{F}_{2}$ and whether plant weight had been affected during irradiation. Alternatively it was to serve as an independently segregating factor, not obtained by environmental induction, to establish whether there were any other unusual features of crosses between $L$ and $S$, and later between $L h$ and $S H$. A white-flowered variety, Stormont Motley, was also used for crosses with $L$ and $S$ after initial crosses had established that $w$ in this variety was at the same locus as the $w$ mutation in $L$. It appears that this locus may be close to the chromosomal regions determining plant weight and $H-h$. Another variety, Mandarin, with purple flowers, gave no association between flower colour and plant weight in the $\mathrm{F}_{2}$ of crosses with $L$ and $S$ respectively and these results are not detailed further.

\section{A GROSS INVOLVING $h$, w AND PLANT WEIGHT}

The growth of the white-flowered $L$ plants, $L w$ (obtained by irradiating the blue-flowered $L$ genotroph, $L W$ ), was first compared with $L W$ and $S W$ (the blue-flowered $S$ genotroph) by growing them and their reciprocal $F_{1}$ 's 
in the field, where 124 plants were all randomised. In the first set in table 1, though the crosses with $L W$ are somewhat lower in plant weight than their parents they are not significantly different from them, and the highly significant differences among the second set, containing the crosses with $S W$ have the pattern expected if $L W$ had been used instead of $L w$. As far as can be judged from these results there are no differences between $L W$ and $L w$ other than at the $w$ locus itself.

$S W$ female was crossed with $L w$ male at Aberystwyth and $10 \mathrm{~F}_{1}$ seeds from

TABLE 1

Mean plant weights $(g)$ of parents and $F_{1} s$ of reciprocal crosses between $\mathrm{Lw}$ and $\mathrm{LW}, \mathrm{Lw}$ and SW. The mean plant weight of the same Lw plants is given in both sets

$\begin{array}{cccc}L w & L w \times L W & L W \times L w & L W \\ 83.5 & 71 \cdot 0 & 74 \cdot 1 & 78 \cdot 8 \\ L w & L w \times S W & S W \times L w & S W \\ 83.5 & 42 \cdot 8 & 39 \cdot 1 & 15 \cdot 3\end{array}$

a single capsule were grown in the Earhart Laboratories, California Institute of Technology, in winter with $10 \mathrm{SW}$ and $10 \mathrm{Lw}$ seeds in a day/night temperature regime of $27^{\circ} / 15^{\circ}$, and a 16 -hour day. The cross was made in this direction because $S \times L \mathrm{~F}_{1}$ has greater genetic instability in plant weight than the $\mathrm{F}_{1}$ of $L \times S . \quad \mathrm{F}_{2}$ families of 10 plants each were grown from six of the $\mathrm{F}_{\mathbf{1}}$ plants. The 55 of the $60 \mathrm{~F}_{2}$ plants which grew were randomised with 29 plants from selfed $L w$ and 30 plants from selfed $S W$ in $27^{\circ} / 15^{\circ}$ and 16-hour day in the Earhart Laboratories in summer.

TABLE $2 a$

Mean plant weights $(g)$ and $\mathrm{W}: \mathrm{w}$ ratios (in $F_{3}$ from segregating families) in the $\mathrm{SW} \times \mathrm{Lw}$ cross

$\begin{array}{crccc} & & & & \text { Ratio } \\ & S W & S W \times L w & L w & W: w \\ \mathrm{~F}_{1} & 9.3 & 15 \cdot 1 & 11.0 & - \\ \mathrm{F}_{2} & 12.4 & 13.9 & 16.0 & 37: 18 \\ \mathrm{~F}_{3} & 13.7 & 38.6 & 59 \cdot 7 & 81: 20\end{array}$

TABLE $2 b$

Mean plant weights $(g)$ of blue- and white-flowered plants in each $F_{2}$ family

\begin{tabular}{lrrrrrrr} 
Family & 1 & 2 & 3 & 4 & \multicolumn{1}{c}{5} & \multicolumn{1}{c}{6} & Mean \\
Blue & 13.7 & 13.7 & 15.4 & 15.4 & 14.6 & 15.6 & 14.73 \\
White & 14.6 & 12.6 & $12 \cdot 2$ & 12.4 & 12.6 & 14.0 & 13.07 \\
Blue-White & -0.9 & 1.1 & 3.2 & 3.0 & 2.0 & 1.6 & 1.66
\end{tabular}

In these environments the plant weight difference between $L w$ and $S W$ in both generations is small (table $2 a$ ). The $\mathrm{F}_{1}$ plants are substantially larger than either parent in the winter, while in the summer the $F_{2}$ plants have the midparent value. This is not due to $F_{1}$ heterosis disappearing in the $F_{2}$ but to a genotype $\times$ environment interaction of the sort described in $L, S, L \times S, S \times L, \mathrm{~F}_{1}$ and $\mathrm{F}_{2}$ plants grown in the summer and winter at Aberystwyth (Durrant, 1971). Table $2 b$ shows that in the $\mathrm{F}_{2}$, in five out of six families, blue-flowered plants are larger than white-flowered plants, and 
the overall difference in plant weight between blue and white is significant (table 2c) when tested against the within-family variation $(\mathrm{P}<0 \cdot 1$ per cent), since a comparison can be made within each family, and when tested against the between-family variation $(\mathrm{P}<\mathrm{l}$ per cent). The variation between blueflowered plants is significantly greater than the variation between whiteflowered plants. This association between flower colour and plant weight

TABLE $2 c$

Analysis of variance of $F_{2}$ plant weights

$\begin{array}{lccc}\text { Item } & & \text { d.f. } & \begin{array}{c}\text { Mean } \\ \text { square }\end{array} \\ \text { Blue-White } & & 1 & 27 \cdot 36 * * * \\ \text { Between families: } & \text { blue } & 5 & 4 \cdot 76 \\ & \text { white } & 5 & 0 \cdot 06 \\ \text { Within families: } & \text { blue } & 31 & 2 \cdot 35 \\ & \text { white } & 12 & 0 \cdot 13\end{array}$

Significance levels in all tables; $* 5$ per cent. $* * 1$ per cent. $* * * 0.1$ per cent.

is in the opposite direction to linkage. It is not apparently due to selection, because in the first place the inviability, or slow germination, of five out of the 60 seeds sown, due probably to the poorer quality of seed ripened under winter conditions, cannot make much difference to the result. Secondly, the deviation of $W: w$ ratio $(37: 18)$ from $3: 1$ has a 20 per cent probability of being due to sampling error, but if it were supposed nevertheless that there were selection against the small blue-flowered genotypes in the $F_{2}$ prior to seed set and the $17 \mathrm{~W}$ plants required to bring the ratio to $3: 1$ exactly were all of the small type, the overall difference in plant weight between blueflowered and white-flowered plants would still remain substantial, 1.04 grams, which is about 30 per cent of the difference in plant weight between the $S W$ and $L w$ parents grown with them.

TABLE $2 d$

Mean plant weights $(g)$ of blue- and white-flowered $F_{2}$ plants selected for high and low plant weight, and their $F_{3}$ families. Also mean plant weights of blue- and white-flowered plants within the segregating $F_{3}$ families

\begin{tabular}{|c|c|c|c|c|c|c|}
\hline & \multicolumn{3}{|c|}{$\begin{array}{l}\text { Selected from blue- } \\
\text { flowered } \mathrm{F}_{2} \text { plants }\end{array}$} & \multicolumn{3}{|c|}{$\begin{array}{l}\text { Selected from white- } \\
\text { flowered } \mathbf{F}_{2} \text { plants }\end{array}$} \\
\hline & High & Low & Mean & High & Low & Mean \\
\hline Selected $F_{2}$ plants & $17 \cdot 4$ & $13 \cdot 3$ & $15 \cdot 3$ & $13 \cdot 7$ & $12 \cdot 0$ & $12 \cdot 8$ \\
\hline $\mathrm{F}_{3}$ families & $42 \cdot 7$ & $41 \cdot 6$ & $42 \cdot 2$ & $34 \cdot 7$ & $23 \cdot 7$ & $29 \cdot 2$ \\
\hline Blue-flowered in $F_{3}$ & $49 \cdot 0$ & $43 \cdot 3$ & $46 \cdot 1$ & - & 一 & - \\
\hline White-flowered in $\mathbf{F}_{3}$ & $30 \cdot 5$ & $31 \cdot 7$ & $31 \cdot 1$ & - & - & - \\
\hline
\end{tabular}

$\mathrm{F}_{3}$ families were grown at Aberystwyth and they were the progeny of the largest and smallest blue-flowered plants and the largest and smallest whiteflowered plants selected from within each of three $\mathrm{F}_{2}$ families, together with some families of $L w$ and $S W$, a total of 336 plants. They were reared in an unheated greenhouse for the first 5 weeks and transplanted into the field in randomised blocks. Under these more normal conditions $L w$ was four times the size of $S W$, and the $\mathrm{F}_{3}$ of the cross was intermediate in weight (table $2 a$ ). The mean plant weights of the selected $F_{2}$ plants and of their progeny separated into the four classes of high and low selection within each flower colour are shown in table $2 d$. 
$F_{3}$ families from white-flowered $F_{2}$ plants are smaller (29.2 grams) than those from blue-flowered (42.2), showing that the colour associated plant weight differences in the $\mathrm{F}_{2}$ are inherited. Selection for plant weight has been effective among the white-flowered $\mathrm{F}_{2}$ plants (in $\mathrm{F}_{3}, 34.7$ for the high selection, 23.7 low selection) but not among the blue-flowered in $\mathrm{F}_{2}$ (in $\mathrm{F}_{3}$, 42.7 and $41 \cdot 6$ ). In fact, over all the data, a greater difference in plant weight is obtained in $\mathrm{F}_{3}$ by selecting for flower colour in $\mathrm{F}_{2}$ than by selecting for plant weight. When the blue- and white-flowered plants are separated within the segregating $\mathrm{F}_{3}$ families (table $2 d$ ) the white-flowered plants are again smaller than the blue-flowered, within the high and low selection lines. All associated plant weight differences are highly significant. This association in the reverse direction to linkage therefore occurs consistently within $F_{2}$ families, and between and within $\mathrm{F}_{3}$ families.

At the time this experiment was done (1959-1963) the $h$ character had not been recognised, but the capsules of all $\mathrm{F}_{3}$ plants were examined later and

TABLE $2 e$

Mean plant weights $(g)$ of four phenotypes in the progeny of Lwh/SWH heterozygotes. $\chi^{2}$ tests on segregations and linkage

\begin{tabular}{|c|c|c|c|c|c|c|}
\hline \multirow[b]{2}{*}{ Weight } & \multirow{2}{*}{$\begin{array}{l}W H \\
38 \cdot 9\end{array}$} & \multirow{2}{*}{$\begin{array}{c}W h \\
43 \cdot 7\end{array}$} & \multirow{2}{*}{$\begin{array}{r}w H \\
28 \cdot 5\end{array}$} & \multirow{2}{*}{$\begin{array}{c}w h \\
23 \cdot 2\end{array}$} & \multicolumn{2}{|c|}{$\begin{array}{c}\text { Mean plant } \\
\text { weight difference }\end{array}$} \\
\hline & & & & & $\begin{array}{c}h-H \\
w-W\end{array}$ & $\begin{array}{c}0.3 \\
-16 \cdot 5 * * *\end{array}$ \\
\hline Number of plants & 22 & 8 & 3 & 3 & & \\
\hline
\end{tabular}

Segregation $H / h ; \quad \chi_{1}^{2}=1.33$

Segregation $W / w ; \chi_{1}^{2}=0.59$

Linkage $w / h ; \quad \chi_{1}^{2}=0.79$

the presence or absence of hairs recorded. In the $\mathrm{F}_{2}$ of crosses between $L$ and $S$ in the absence of $w$ a strong and highly significant association was obtained previously between $h$ and plant weight in the direction of linkage (Durrant and Nicholas, 1970) and the purpose was to see whether $h$ was associated with plant weight and $w$ in the $\mathrm{F}_{3}$ here. Of the $12 \mathrm{~F}_{2}$ plants from which $\mathrm{F}_{3}$ families were grown, four were homozygous $h h$, four were homozygous $H H$, with different combinations of $w$ and $W$, two were heterozygous $H h$ with homozygous $w w$, i.e. $w h / w H$, one in high and one in low selection, and two were complete heterozygotes, wh/WH, also one in high and one in low selection. Plants of the eight families of the two homozygous types embraced unequally progeny of $F_{2}$ flower colour selection, $F_{2}$ plant weight selection and colour segregants within $\mathrm{F}_{3}$ families, making a comparison between the two homozygous types unreliable, but the unweighted mean plant weights of $H$ and $h$ plants in $\mathrm{F}_{3}$ worked out to 37.6 and 37.5 grams respectively, i.e. no association between $h$ and plant weight. The mean plant weight of $H$ and $h$ plants with homozygous ww had low values, 23.9 and 26.0 respectively, because of the association of $w$ with plant weight, but they were not significantly different.

The mean plant weight of $H$ and $h$ plants with segregating $W / w$ are given in table $2 e$, which shows despite the small number of plants that the association of plant weight with $w$ is highly significant, giving a difference of 16.5 grams which is 38 per cent of the difference between the parental plant weight in $\mathrm{F}_{3}$ (table $2 a$ ), whereas with $h$ it is negligible. There is no evidence 
of abnormal segregations, nor of linkage between $w$ and $h$. In table $2 a$ the overall $W: w$ ratio in $\mathrm{F}_{3}$ is not significantly different from $3: 1$, deviating in the opposite direction to that in $\mathrm{F}_{2}$, which together give a $W: w$ ratio of $3 \cdot 05: 1$.

The conclusions are that in this cross and under these conditions, $w$ is associated with plant weight in the opposite direction to linkage, but $h$ shows no association with plant weight although in previous experiments in the absence of $w$ it had shown strong association in the direction of linkage. $w$ and $h$ are not apparently linked and they segregate normally.

\section{RECIPROCAL CROSSES INVOLVING $h, w$ AND PLANT WEIGHT}

These crosses were basically the same as the one in the previous section, except that here the reciprocal crosses were made, $L w h \times S W H$ and $S W H \times L w h$, and crosses were also made reciprocally between $L w h$ and $L W h$,

TABLE $3 a$

Mean plant weights $(g)$ of parents and $F_{2}$ 's of reciprocal crosses between Lwh and SWH

$\begin{array}{cccc}\text { Lwh } & \text { Lwh } \times S W H & S W H \times L w h & S W H \\ 37.6 & 31.2 & 33.7 & 21.5\end{array}$

TABLE $3 b$

Mean plant weights $(g)$ of blue-flowered and whiteflowered plants in the $F_{2}$ of crosses between $L W$ and $L w$

$\begin{array}{lccr} & \text { Blue }(W) & \text { White }(w) & w-W \\ L W \times L w & 38.9 & 39.3 & 0.4 \\ L w \times L W & 39.6 & 37.1 & -2.5\end{array}$

but the environment was different. The parents were grown in the Earhart Laboratories with $19^{\circ} / 11^{\circ}$ day/night temperatures, 16-hour day and Hoagland's solution. The $\mathrm{F}_{1}$ was transplanted into the field after 5 weeks in a heated greenhouse at Aberystwyth in 1963. The $F_{2}$ was grown the following year out-of-doors from sowing, except for the third week when there was room in a cool greenhouse, and later transplanted into the field. The plant weights, flower colour and capsule scores are summarised in table 3.

The parental difference in the $\mathrm{F}_{2}$ (table $3 a$ ) was large though not so large as would be obtained in an optimum environment. The reciprocal $\mathrm{F}_{2}$ 's do not differ significantly from one another nor from the midparent value. Table $3 b$ shows that in the reciprocal crosses between large blue and large white plants, $L W$ and $L w$, there is no significant association of flower colour with plant weight; apparently only the $w$ locus differs between them. In table $3 c$ the $\chi^{2}$ 's show that $h$ and $w$ segregate normally and there is no evidence of linkage between them. The association between plant weight and $W / w$ segregants in the $S W H \times L w h$ cross is again in the opposite direction to linkage but only significant on a single tail test: in the reciprocal cross there is no association of $W / w$ segregants with plant weight. Conversely in the $L w h \times S W H$ cross $H / h$ segregants are strongly and highly significantly associated with plant weight in the direction of linkage, but it is less strong though still significant in the $S W H \times L w h$ cross. Consequently among the 
TABLE $3 c$

Mean plant weights $(g)$ of four phenotypes in the $F_{2}$ of crosses between Lwh and $\mathrm{SWH}$. $\chi^{2}$ tests on segregations and linkages

$\begin{array}{lccccc} & W H & W h & w H & w h & \begin{array}{c}\text { Mean plant } \\ \text { weight difference }\end{array} \\ S W H \times L w h & 27 \cdot 0 & 34 \cdot 3 & 23 \cdot 6 & 31 \cdot 1\left\{\begin{array}{ccc}h-H & 7 \cdot 4^{*} \\ w-W & -3 \cdot 4\end{array}\right. \\ \begin{array}{lcccc}L w h \times S W H & 30 \cdot 8 & 40 \cdot 4 & 28 \cdot 1 & 42 \cdot 8\end{array}\left\{\begin{array}{ccc}h-H & 12 \cdot 1^{* *} \\ w-W & -0 \cdot 2\end{array}\right. \\ \text { Number of plants } & 85 & 37 & 37 & 12\end{array}$

Segregation $H / h ; \quad \chi_{1}^{2}=1 \cdot 22$

Segregation $W / w ; \quad \chi_{1}^{2}=1 \cdot 22$

Linkage $w / h ; \quad \chi_{1}^{2}=0.58$

crosses there is an inverse relation between the association of $h$ with plant weight in the direction of linkage and of $w$ with plant weight in the opposite direction to linkage. The crosses which are in the same direction, $S W H \times L w h$, give the more similar results.

\section{OUtcrosses INVOLving $w, h$ AND PLANT WEIGHT TO ANOTHER VARIETY}

Crosses were made in the field at Aberystwyth between three genotrophs of Stormont Cirrus and another variety, Stormont Motley $(K)$, which has white flowers and hairless capsules, i.e. $K / K, w / w, h / h . \quad L$ and $S$ parents used for crossing were grown in a heated greenhouse for the first 5 weeks before

TABLE $4 a$

Mean plant weights $(g)$ of parental lines, used in outcrossing, grown with the $F_{2}$ plants given in table $4 b$

$\begin{array}{cccc}L & P l & S & K \\ 29 \cdot 8 & 20 \cdot 7 & 9 \cdot 9 & 10 \cdot 0\end{array}$

TABLE $4 b$

Mean plant weights $(g)$ of the phenotypes in the $F_{2}$ of crosses between $\mathrm{L}$ and $\mathrm{K}$, $\mathrm{S}$ and $\mathrm{K}, \mathrm{Pl}$ and $\mathrm{K}$. Numbers of plants are in brackets. $\chi^{2}$ tests on segregations and linkages

\begin{tabular}{|c|c|c|c|c|c|c|}
\hline \multirow{3}{*}{$\begin{array}{l}L W \times K w \\
K w \times L W\end{array}$} & \multirow{3}{*}{$\begin{array}{l}W H \\
-\end{array}$} & $W h$ & $w H$ & $w h$ & \multicolumn{2}{|c|}{$\begin{array}{c}\text { Mean plant } \\
\text { weight difference }\end{array}$} \\
\hline & & $15 \cdot 1$ & - & 14.5 & $w-W$ & -0.6 \\
\hline & & & - & & & \\
\hline $\begin{array}{l}P l W H \times K w h \\
K w h \times P l W H\end{array}$ & $\begin{array}{l}14 \cdot 4 \\
(16)\end{array}$ & $\begin{array}{l}17 \cdot 2 \\
(7)\end{array}$ & $\begin{array}{l}15 \cdot 1 \\
(12)\end{array}$ & $\begin{array}{l}17 \cdot 2 \\
(6)\end{array}$ & $\begin{array}{c}h-H \\
w-W\end{array}$ & $\begin{array}{l}2 \cdot 4 \\
0 \cdot 7\end{array}$ \\
\hline \multirow[t]{2}{*}{$\begin{array}{l}S W H \times K w h \\
K w h \times S W H\end{array}$} & $\begin{array}{l}12 \cdot 7 \\
(29)\end{array}$ & $\begin{array}{l}13 \cdot 5 \\
(9)\end{array}$ & $\begin{array}{l}13 \cdot 5 \\
(7)\end{array}$ & $\begin{array}{l}13 \cdot 5 \\
(1)\end{array}$ & $\begin{array}{l}h-H \\
w-W\end{array}$ & $\begin{array}{l}0 \cdot 4 \\
0 \cdot 4\end{array}$ \\
\hline & & \multicolumn{2}{|c|}{$L$ and $K$} & $P l$ and $K$ & \multicolumn{2}{|c|}{$S$ and $K$} \\
\hline \multirow{2}{*}{\multicolumn{2}{|c|}{ Segregation $H / h$; }} & \multicolumn{2}{|c|}{ - } & 0.98 & \multicolumn{2}{|c|}{$0 \cdot 26$} \\
\hline & & \multicolumn{2}{|c|}{$0 \cdot 07$} & $7 \cdot 81 * *$ & \multicolumn{2}{|c|}{$1 \cdot 42$} \\
\hline Linkage $w$ & & \multicolumn{2}{|c|}{-} & 0.46 & \multicolumn{2}{|c|}{0.24} \\
\hline
\end{tabular}


transplanting but $P l$ and $K$ were kept outside. The $\mathrm{F}_{1}$ and $\mathrm{F}_{2}$ were grown in successive years out-of-doors from sowing and transplanted into the field.

The mean plant weights of the parental lines grown with the $\mathrm{F}_{2}$ are given in table $4 a . \quad L$ is three times the weight of $S, P l$ is intermediate and $K$ has the same plant weight as $S$ although it is a much taller plant. The $\chi^{2}$ 's in table $4 b$ show normal segregations for $H / h$ and $W / w$ except in the cross with $P l$, where $W: w$ has a $1: 1$ ratio instead of a $3: 1$ ratio, and no linkage between $h$ and $w$ in either of the crosses where both segregate. There is no significant association anywhere between $w$, or $h$, with plant weight. Comparison of the plant weights in tables $4 a$ and $4 b$ shows that the $L$ and $S$ crosses with $K$ are apparently reverting towards the value of the $P l$ cross with $K$ which, if a true reversion of the induced $L$ and $S$ changes, is probably directly due to chromosomal factors in $K$ (Durrant, 1972).

\section{Discussion}

The three characters have the following features in common. They are associated in induction, in inheritance and in the products of crosses but the associations are not complete and most combinations can be obtained either by altering the environment or by crossing. Plant weight and DNA amount have an apparently continuous range of genetic variation and $H h$ heterozygotes have several levels of activity. They are unstable when heterozygous and the range of variation of each is due probably to different levels of activity of a particular region rather than to a multigenic system whose factors are distributed over the chromosomes. None of the $\mathrm{F}_{2}$ genetic variation need necessarily be due to classical segregation and recombination. The environmental induction of $L$ and $S$ is symmetrical about $P l$ in plant weight and DNA amount as though equal and opposite changes are induced (Durrant, 1962a, 1971). Factors for the three characters are probably together on one chromosome rather than dispersed over the chromosomes.

The primary change may be in DNA amount, arising from an induced increase or decrease in number of reiterated sequences, or from some other unknown chromosomal re-arrangement, which alters the coiling, heterochromatisation or structure of the chromosomes and the activity of the genes in the region. A nuclear factor and a cytoplasmic factor are required for induction of changes in DNA amount (Durrant and Timmis, 1973). If the nuclear factor were in a region over which heterochromatisation spreads, the induced changes could be maintained in later generations in the absence of inducing environments. In the heterozygote of crosses between $L$ and $S$ their separate stable equilibria are destroyed and the system breaks down, throwing up, perhaps in a disorderly manner, combinations of heterochromatic sub-regions.

If $L$ and $S$ plant weights were determined by two alleles at one locus and $H$ and $h$ are two alleles of another locus completely linked to the first, if $H$ is fully dominant to $h$ (or $H h$ heterozygotes are scored as $H$ ) but neither $L$ or $S$ is dominant in plant weight to the other, the difference between the mean plant weights of $H$ and $h$ plants in the $\mathrm{F}_{2}$ of a cross $L h \times S H$, or its reciprocal, would be two-thirds of the parental difference. If $L$ were completely dominant in plant weight to $S$ the difference in the $\mathrm{F}_{2}$ would be one-third of the parental difference. In the second experiment (table 3) the $h-H$ plant weight difference (12.1 grams) in the $\mathrm{F}_{2}$ of $L w h \times S W H$ cross is 75 per cent 
of the $L-S$ parental difference in plant weight of $16 \cdot 1$ grams. This is comparable with the 67 per cent expected for no dominance in plant weight and complete linkage, and much in excess if the partial dominance of $L$ in this $\mathrm{F}_{2}$ is taken into account. On these assumptions plant weight is completely linked to $h$. In the reciprocal cross, $S W H \times L w h$, the $h-H$ plant weight difference is 46 per cent of the parental difference which, allowing for dominance, also infers complete linkage. The concept of physical linkage is valid even though the $F_{2}$ genetic variation may be due to instability rather than mendelian segregation.

In the $F_{2}$ and $F_{3}$ of the first experiment $w$ is associated with plant weight in the reverse direction to linkage. High-frequency crossing over at the two-strand stage is unlikely and the $w$ mutation obtained by $\mathrm{Co}^{60}$ radiation must be an alteration in controlling elements of $w$ rather than the $w$ locus

TABLE 5

Mean plant weight differences $(g)$ between $\mathrm{H}$ and $\mathrm{h}$ plants, $\mathrm{W}$ and $\mathrm{w}$ plants, in the $F_{2}$ or $F_{3}$ of separate crosses, expressed as percentages of the parental mean plant weight differences (L-S). $\mathrm{h}$ plants are larger than $\mathrm{H}$ and show "positive linkage". $\mathrm{w}$ plants are smaller than W and show" negative linkage ".

$\begin{array}{lrr} & h-H & w-W \\ S W H \times L w h & 0.7 & -38.4 \\ S W H \times L w h & 46.0 & -21 \cdot 1 \\ L w h \times S W H & 75.2 & -1 \cdot 2\end{array}$

itself which is presumably near to the region covering the plant weight and $h$ factors. The amounts of "negative linkage" of $w$, and of "positive linkage" of $h$, with plant weight are inversely related in the three crosses in tables $2 e$ and $3 c$. The plant weight differences in the $\mathrm{F}_{2}$ or $\mathrm{F}_{3}$ for $h-H$ and $w-W$ are brought together and standardised in table 5 as percentages of the differences in plant weight between the $L$ and $S$ parents in the different crosses, and they give virtually a straight-line relation. But they are only three points and statistically do not permit much interpolation.

Given the right environmental conditions, the amounts of DNA in $L$ and $S$ revert towards the intermediate amount found in the $P l$ genotroph (Durrant and Jones, 1971) and it is likely that they revert in the $L / S$ heterozygote as well, decreasing in the $L$ chromosome and increasing in the $S$ chromosome. Heterochromatisation, or its loss, or other chromosomal changes brought about by heterozygosity, may occur in different parts of the region depending on the environment and direction of the cross. Because of the symmetry of the induced $L$ and $S$ changes the influence of each chromosome on its homologue in the heterozygote is mutual, not unidirectional as, for example, in paramutation of the $R$ locus in maize, but in the appropriate environment at one extreme $L W$ and $S w$ associations are expressed by the respective chromosomes which are at random with regard to $H$ and $h$, in other environments at the other extreme $L h$ and $S H$ association are expressed which are at random with $W$ and $w$, and combinations of both in intermediate environments. This process entails three events. Interaction between homologous chromosomes producing changes in both. Interaction 
along each homologue giving relatively stable segments. Modulation along the chromosomes by the intracellular environment, stabilisation at one point allowing a more random association of factors at another, or lowers the probability of further changes. Even if only a portion of the 16 per cent difference in amount of DNA between $L$ and $S$ was in this region this could represent a difference of several orders of magnitude in number of reiterated sequences, probably causing large adjustments in heterochromatisation in the region, which may be the reason for the occurrence of the recessives $h$ and $w$ in the $L$ genotroph. When the homologous chromosomes are brought together in the heterozygote, which has an intermediate amount of DNA, heterochromatisation of the segment containing $w$ is transferred to its homologue over a number of cell divisions wherein a balance is achieved by the reduction in one chromosome and an increase in the other, giving at once "negative linkage" with plant weight, i.e. $W \rightarrow w$ and $w \rightarrow W$ on the respective chromosomes. The adjustment at $w$ permits random association elsewhere. In other environments segments containing the plant weight and $h$ factors are unchanged allowing random association of $w$. The region as a whole eventually reaches a balance, but the segments would have participated in different ways depending upon the environment. Crosses in the other phase would help confirm this interpretation.

In the outcrosses of $L, S$ and $P l$ to another variety, Stormont Motley $(K)$, there is no significant association of $h$ or $w$ with plant weight (table 4). This is one of the varieties which apparently destroys, or reverts, the induced $L$ and $S$ plant weight differences when $L$ and $S$ are crossed with it (Durrant, 1972). There is no interplay here between two symmetrically induced types as in $L / S$ heterozygotes but a directive effect of $K$. Among all the crosses described the only significant deviation from a 3:1 ratio occurs in the cross of $K$ with $P l$ where there is a a highly significant excess of $w$ plants (deviation $\chi_{1}^{2}=7 \cdot 81$ ), and two reciprocals giving similar results (heterogeneity $\chi_{1}^{2}=0 \cdot 42$ ). Apparently this is a further directed change by $K$ which converts with high-frequency $W$ to $w$ in the plastic genotroph. A cytoplasmic factor could participate because $W: w$ is close to a $1: 1$ ratio which would be given by a $W \rightarrow w$ change being maintained through the male gamete only, or female gamete only.

The influence of one chromosome on the number of reiterated gene sequences of its homologue has been measured at the compound $b b$ locus in Drosophila melanogaster composed of genes for ribosomal RNA by Boncinelli et al. (1972). The number of genes for rRNA on one chromosome of a fly showing the bobbed character increases or decreases inversely with the number of genes present at the same locus on the homologous chromosome. Other chromosomal changes which have some common features to those described here are the following. In female mammals either one $\mathrm{X}$ chromosome or the other can be inactivated by heterochromatisation (Lyon, 1961). Environmentally induced changes in Nicotiana rustica are unstable in the $\mathrm{F}_{\mathbf{1}}$ of crosses between them (Perkins, Eglington and Jinks, 1971). Instability at the pal locus in Antirrhinum majus is influenced by temperature and another gene (Harrison and Fincham, 1964, 1968). Paramutation at the $R$ locus in maize is influenced by neighbouring chromosomal regions (Brink, 1969). Paramutation at the sulf locus in Lycopersicum esculentum is due to the heterochromatisation of the locus (Hagemann and Snoad, 1971). Mottled aleurone colour in maize is dependent upon whether the $R$ allele is trans- 
mitted by the male or female gamete (Kermicle, 1970). These are interactions between homologous chromosomes, parts of chromosomes or between these and environments inducing at least semi-permanent changes in the chromosomes and it is credible that chromosomal regions in flax in which changes are induced by the environment can also be altered by homologous chromosomes differing from them, or by changes in nearby segments.

Acknowledgments. - I am grateful to Professor Anton Lang for facilities provided at the Earhart Laboratories, Biology Division, California Institute of Technology, Pasadena. The work at the Earhart Laboratories was supported by a grant (G-17483) of the National Science Foundation. I am also grateful to Mrs Margaret K. Genthe for her technical assistance.

\section{REFERENCES}

BONCINELli, E., GRAZIANi, F., POLITO, L., MALVA, C., AND RITOSSA, F. 1972. rDNA magnification at the bobbed locus of the $\mathrm{Y}$ chromosome in Drosophila melanogaster. Cell Diff., 1 , 133-142.

BRINK, R. A. 1969. Abnormal chromosome 10 and $R$ paramutation in maize. Mut. Res., 8, 285-302.

DURRANT, A. 1962a. The environmental induction of heritable change in Linum. Heredity, $17,27-6 \mathrm{I}$.

DURRANT, A. 1962b. Induction, reversion and epitrophism of flax genotrophs. Nature, 204, 1302-1304.

DURRANT, A. 1971. The induction and growth of flax genotrophs. Heredity, 27, 277-298.

DURRANT, A. 1972. Studies on reversion of induced plant weight changes in flax by outcrossing. Heredity, 29, 71-81.

DURRANT, A., AND JONES, T. W. A. 1971. Reversion of induced changes in amount of nuclear DNA in Linum. Heredity, 27, 431-439.

DURRANT, A., AND NICHOLAS, D. B. 1970. An unstable gene in flax. Heredity, 25, 513-527. DURRANT, A., AND TIMMIs, J. N. 1973. Genetic control of environmentally induced changes in Linum. Heredity. (In Press.)

Evans, G. M. 1968. Nuclear changes in flax. Heredity, 23, 25-38.

EVANS, G. M., DURRANT, A., AND REES, H. 1966. Associated nuclear changes in the induction of flax genotrophs. Nature, 212, 697-699.

hagemanN, R., AND SNOAD, B. 1971. Paramutation (somatic conversion) at the sulfurea locus of Lycopersicum esculentum. V. The localisation of sulf. Heredity, 27, 409-418.

HARRISON, B. J., AND FINCHAM, J. R. s. 1964. Instability at the pal locus in Antirrhinum majus. 1. Effects of environment on frequencies of somatic mutation and germinal mutation. Heredity, 19, 237-258.

HARRISON, B. J., AND FINCHAM, J. R. S. 1968. Instability at the pal locus in Antirrhinum majus. 3. A gene controlling mutation frequency. Heredity, 23, 67-72.

Kermicle, J. L. 1970. Dependence of the $R$-mottled aleurone phenotype in maize on mode of sexual transmission. Genetics, 66, 69-85.

LYON, M. F. 1961. Sex chromatin and gene interaction in the mammalian X-chromosome. Am. F. Hum. Genet., 14, 135-148.

MCLELLAN, J. C., AND DURRANT, A. 1973. Instability of $H$ heterozygotes in flax genotrophs. Heredity, 30, 63-71.

PERKINS, J. M., EGLINGTON, E. G., AND Jinks, J. L. 1971. The nature of the inheritance of permanently induced changes in Nicotiana rustica. Heredity, 27, 441-457. 